\title{
Nonequilibrium Thermodynamics Based on the Distributions Containing Lifetime as a Thermodynamic Parameter
}

\author{
Vasiliy Vasiliy Ryazanov \\ Kiev Institute for Nuclear Research, National Academy of Sciences of Ukraine, prospekt Nauki 47, \\ 252028 Kiev, Ukraine \\ Correspondence should be addressed to Vasiliy Vasiliy Ryazanov, vryazan@kinr.kiev.ua
}

Received 7 June 2011; Revised 3 August 2011; Accepted 5 August 2011

Academic Editor: L. De Goey

Copyright ( $) 2011$ Vasiliy Vasiliy Ryazanov. This is an open access article distributed under the Creative Commons Attribution License, which permits unrestricted use, distribution, and reproduction in any medium, provided the original work is properly cited.

To describe the nonequilibrium states of a system, we introduce a new thermodynamic parameter-the lifetime of a system. The statistical distributions which can be obtained out of the mesoscopic description characterizing the behaviour of a system by specifying the stochastic processes are written down. The change in the lifetime values by interaction with environment is expressed in terms of fluxes and sources. The expressions for the nonequilibrium entropy, temperature, and entropy production are obtained, which at small values of fluxes coincide with those derived within the frame of extended irreversible thermodynamics. The explicit expressions for the lifetime of a system and its thermodynamic conjugate are obtained.

\section{Introduction}

In papers [1-3], the linear nonequilibrium thermodynamics (LIT) was developed which found its application in the variety of physical problems. But the linear irreversible thermodynamics possesses a number of restrictions, which present certain obstacles for adequately describing such phenomena as the propagation and absorption of ultrasound in liquids, density profile of shock waves in gases, and so forth. The attempts to overcome these difficulties led to creating the extended irreversible thermodynamics (EIT) [4]. If in the frame of LIT [1-3] the local entropy density is merely a function of conserving quantities such as local energy and mass densities, in EIT a set of additional variables is added thereto, which are chosen as densities of fluxes in a system (nonconserving dissipative values). Phenomenological laws appear to be nonstationary equations of motions, the entropy flux-containing nonlinear terms; the velocity of propagation of perturbations is finite in the contrast to the infinite velocity (e.g., of propagating heat waves) predicted by classical thermodynamics. The Fourier law $\vec{q}=-\lambda \vec{\nabla} T$ (where $\vec{q}$ is the heat flux, $\lambda$ is the heat conductivity coefficient, and $T$ is absolute temperature) of the classical LIT is replaced by the Maxwell-Cattaneo equation

$$
\vec{q}=-\lambda \vec{\nabla} T-\frac{\tau_{q} \partial \vec{q}}{\partial t}
$$

where $\tau_{q}$ is the time of the flux correlation.

Characterizing the nonequilibrium state by means of an additional parameter related to the deviation of a system from the equilibrium (field of gravity, electric field for dielectrics, etc.) was used in [5]. In the present paper, it is suggested the new choice of such an additional parameter as the lifetime of a physical system, which is defined as a first-passage time till the random process $y(t)$ describing the behaviour of the macroscopic order parameter of a system (energy, e.g.,) reaches its zero value. The lifetime is thus a random process which is slave (in terms of the definitions of the theory of random processes [6]) with respect to the master process $y(t)$,

$$
\Gamma_{x}=\inf \{t: y(t)=0\}, \quad y(0)=x>0 .
$$

The characteristics of $\Gamma$ depend on those of $E=y(t)$. It is important to render firstly a physical interpretation to 


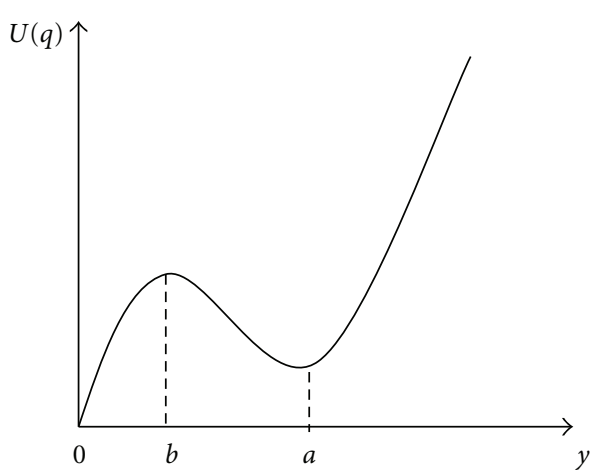

FIgURE 1: The shape of the potential of a system $U(y)$ in the potential well model. The point $y=a$ is the potential well minimum; the barrier in the point point $y=b$ separates the potential well from the zero minimum of $y$.

the definition (2). So, the lifetime is related to the period of stable existence of a system, and its time of dwelling within the homeokinetic plateau whose distribution was related in [7] to the entropic and information parameters of a system, its response to internal and external influences, and its stability and adaptation facilities. Lets show this relation using an illustrative example. Let the behaviour of a system be described by the potential $U(q)$ (Figure 1) having some "potential well" at $\mathrm{y}>b$ and a minimum in the point $y=a$.

The major part of the living time the system dwells within the vicinity of the point a. The time of trespassing by the system value $y=b$ can be considered the finishing of the time of stable existence $T_{\text {stab }}$. Thus during a small time $t_{b 0} \ll T_{\text {stab }}$, the system will roll down to the point $y=0$. The lifetime $\Gamma=T_{\text {stab }}+t_{b 0} \approx T_{\text {stab }}$. Real systems are characterized by much more complicated behaviour, but the dependence $\Gamma \approx T_{\text {stab }}$ seems to hold (with exception of the cases of phase transitions, chaotic regimes, etc.). The states of a system within the homeokinetic plateau are characterized by the mutual compensation of the entropic effects related to the energy dissipation and by the effects of negative entropy determined by the existence of the negative feedbacks. When the system exits out of the limits of the plateau, unstable structures and sharp qualitative changes in the behaviour of the system arise.

The lifetime seems to be a value of more general character than the fluxes are (this fact being the prerequisite of formulating a theory which would include the EIT as its partial case) and, moreover, it seems to be more conservative (that is, the lifetime in this respect stands closer to the ordinary conserving values). The history of a system is the succession of repeating "busy periods"-lifetimes in the sense of the definition (2), and idle periods (like it is the case of the queues systems) which are marked by the observable events of lifetime finishing and system regenerating. The internal time in the system is related to the existence of the nonzero number of elements within. The principal consideration grounding the introduction of the lifetime as a physical parameter is an empirical fact of all systems having limited time of existence. Of interest seems to be applying this notion to the biological objects, for example, investigating the living time of an organism.
Very close to the scope of the present work stand the papers $[8-12]$. In the papers $[8,9]$, the transition of a system from a given initial state to the final state is supposed to take place within a fixed time gap. In the papers [10-12], a microscopic treatment of the decay rate or escape rate out the repeller which is the inverse of the lifetime of the system is performed, which can be readily related to the first-passagetime problems $[6,13]$. But the escape time and escape rate definitions of [10-12] differ from those of the lifetime and its conjugate $\gamma$ used in our paper.

In the present paper, we provide the statistical foundations for the thermodynamic relations and introduce the distribution for the lifetime of a system (Section 2). The main assumptions, namely, concerning the explicit lifetime distribution and the relation between its thermodynamic conjugate and the fluxes are given in the Sections 3 and 4. The explicit expressions for the temperature, entropy, and entropy production are written down and the stability analysis is performed in Section 4. In the Conclusion, various aspects of proposed method for describing complex systems are treated.

\section{Lifetime Distributions}

Let us consider a macroscopic observable $E$ (it might be something different from energy as well) being distributed with function $P(E)=p_{E}(x)$. Using the maximumentropy inference $[14,15]$, one can reconstruct out of the macroscopic distribution $P(E)=p_{E}(x)$ the microscopic probability density in the phase space $z$. The constructing of Gibbs microcanonic distribution corresponds to imposing the additional condition of the equiprobable distribution of all possible microstates. This "maxent" (i.e., related to the maximum-entropy principle) procedure corresponds [16] to the process of coarsening and means physically the loss of information about further details of the behaviour of a system. Let us suppose that bringing a system in the nonequilibrium state violates the equiprobable distribution of equilibrium. We thus introduce a novel parameter $\Gamma(z)$ supposing its observability. We can introduce as well the cells of the extended phase space with constant values of the set $(E, \Gamma)$ within (instead of the cells with constant values of $E)$. The structure factor $\omega(E)$ is thus replaced by $\omega(E, \Gamma)-$ the volume of the hyperspace containing given values of $E$ and $\Gamma$. If $\mu(E, \Gamma)$ is the number of states in the phase space which have the values of $E$ and $\Gamma$ less than given numbers, then $\omega(E, \Gamma)=d^{2} \mu(E, \Gamma) / d E d \Gamma$. It is evident that $\int \omega(E, \Gamma=y) d y=\omega(E)$. The number of phase points between $E, E+d E$ and $\Gamma, \Gamma+d \Gamma$ equals $\omega(E, \Gamma) d E d \Gamma$. We make use now of the principle of equiprobability applied to the extended cells $(E, \Gamma)$. The coarse-grained density representing the growth of the information entropy [16] is merely one of possible ways of introducing irreversibility into the statistical physics. The same procedure was performed in [17], where the systematic loss of information appears by choosing the appropriate operator and eliminating the initial correlations. This approach corresponds to the subjectivistic interpretation $[14,15]$ of the statistical physics. Another approach $[10,11,18,19]$ corresponds to the objectivistic 
interpretation and considers the stochasticity as being the inherent property of the phase space. The lifetime concept is closer to this latter interpretation. From the mathematical viewpoint, one could trace the differences between these two concepts from the differences in constructing the space of elementary events (which in general need not coinciding with the space of coordinates and momenta of all particles as the Gibbs statistics implies).

The standard procedure (e.g., $[16,20]$ ) allows one to write down the relation between the distribution density $P(E, \Gamma)=p_{E \Gamma}(x, y)$ and microscopic (coarse-grained) density $\rho(z ; E, \Gamma)$

$$
P(E, \Gamma)=p_{E \Gamma}(x, y)=\int \delta(E-E(z)) \delta(\Gamma-\Gamma(z)) \rho(z ; E, \Gamma) d z,
$$

where

$$
P(E, \Gamma)=p_{E \Gamma}(x, y)=\left.\rho(z ; E, \Gamma) \omega(E, \Gamma)\right|_{E=x, \Gamma=y} .
$$

Lets introduce the conditional distribution density

$$
P(\Gamma \mid E)=p_{\Gamma}(y \mid x)=\frac{p_{E \Gamma}(x, y)}{p_{E}(x)}=\frac{P(E, \Gamma)}{P(E)},
$$

where

$$
P(E)=p_{E}(x)=\int_{0}^{\infty} \rho(z ; E, \Gamma=y) \omega(E, y) d y .
$$

Now we make an assumption about the form of $\rho(z ; E, \Gamma)$

$$
\rho(z ; E=x, \Gamma=y)=\frac{\exp \{-\beta x-\gamma y\}}{Z(\beta, \gamma)},
$$

where

$$
\begin{aligned}
Z(\beta, \gamma) & =\int \exp \{-\beta x-\gamma y\} d z \\
& =\iint d x d y \omega(x, y) \exp \{-\beta x-\gamma y\}
\end{aligned}
$$

is the partition function and $\beta$ and $\gamma$ are Lagrange multipliers satisfying the equations for the averages

$$
\langle E\rangle=-\left.\frac{\partial \ln Z}{\partial \beta}\right|_{\gamma}, \quad\langle\Gamma\rangle=-\left.\frac{\partial \ln Z}{\partial \gamma}\right|_{\beta} .
$$

We did not concretize yet the parameter $\Gamma(z)$. Now let it be the lifetime of a system (2). Introducing $\Gamma$ means effective account for more information than merely in linear terms of the canonical distribution.

The linearity of the exponent in (7) allows one to relate the expression for the partition function $Z(\beta, \gamma)$ with the
Lagrange transforms of the macroscopic distributions $P(x)$, $P(\Gamma \mid x), P(\Gamma, x)$

$$
\begin{aligned}
E\left(e^{-\theta x}\right) & \equiv Q\left(e^{-\theta}\right)=\int e^{-\theta x} P(x) d x, \\
E\left(e^{-s \Gamma(x)}\right) & \equiv L(x, s)=\int e^{-s y} P(\Gamma=y \mid x) d y, \\
G(\theta, s) & \equiv \int e^{-\theta x-s y} P(E=x, \Gamma=y) d x d y \\
& =L\left(-\frac{\partial}{\partial \theta}, s\right) Q\left(e^{-\theta}\right),
\end{aligned}
$$

where $E(\cdots)$ means averaging. The expressions (10)-(13) can be easily obtained explicitly using the known model of the process $y(t)$ (see Appendix A).

From the expressions (4), (12), and (7)-(8), it is easy to derive the relation between microdynamics (containing in the partition function $Z(\beta, \gamma)$ ) and the Laplace transforms of the macrovariable and its lifetime

$$
G(\theta, s) \equiv \frac{Z(\beta+\theta, s+\gamma)}{Z(\beta, \gamma)} .
$$

Let us choose some reference point $\left(\beta_{0}, \gamma_{0}\right)$ in the space of the Lagrange parameters. If the macroscopic model holds for this point, one can get (using (13)) the value of the nonequilibrium partition function for the other point $(\beta, \gamma)$,

$$
Z(\beta, \gamma)=Z\left(\beta_{0}, \gamma_{0}\right) G\left(\beta-\beta_{0}, \gamma-\gamma_{0}\right) .
$$

The value $Z\left(\beta_{0}, \gamma_{0}\right)$ is merely a number characterizing the "bound part" of the entropy of the microscopic motion. The multiplier $G$ corresponds to the free information derivable when concretizing the stochastic process [21].

\section{Generalized Lifetime Thermodynamics}

If $\gamma=0$ and $\beta=\beta_{0}=\left(k_{B} T_{\mathrm{eq}}\right)^{-1}$, where $k_{B}$ is the Boltzmann constant and $T_{\mathrm{eq}}$ is the equilibrium temperature, then the expressions (7)-(8) yield the equilibrium Gibbs distribution. One can thus consider (7)-(8) as a generalization of the Gibbs statistics to cover the nonequilibrium situation. Such physical phenomena as the metastability, phase transitions, and stationary nonequilibrium states are known to violate the equiprobability of the phase space points. The value $\gamma$ can be regarded as a measure of the deviation from the equiprobability hypothesis. In general, one might choose the value $\Gamma$ as a subprocess of some other kind as chosen above.

Let us suppose that the process $y(t)=E$ is the energy of a system (equivalently one could choose the particle number, pulse, and so forth but for the present paper we shall restrict ourselves with the heat conductivity problem). Further detalization will require the concretization of the $\Gamma$ distribution and the interpretation of the Lagrange parameters $\beta$ and $\gamma$. The Lagrange parameter $\beta$ is supposed to be (likewise the equilibrium Gibbs statistics)

$$
\beta=\frac{1}{k_{B} T},
$$


where $T$ is the average (over the body volume) local equilibrium temperature. Since at fluxes $\vec{q} \neq 0$ the temperature is not the same all over the bulk of the body, one can define $T$ in a system with volume $V$ as the volume average, that is, $T=V^{-1} \int_{V} T(r, t) d r$ (the same definition was used in [22]). Of course, the thermodynamic description itself is supposed to be already coarse-grained. To get the explicit form of the $\Gamma$ distribution, we shall use the general results of the mathematical theory of phase coarsening of the complex systems [23] (Appendix B), which imply the following distribution of the lifetime for coarsened random process:

$$
p_{\Gamma}(y)=\Gamma_{0}^{-1} \exp \left\{-\frac{y}{\Gamma_{0}}\right\} .
$$

The values $\Gamma_{0}$ are averaging of the residence times and the degeneracy probabilities over stationary ergodic distributions (in our case, Gibbs distributions). The physical reason for the realization of the distribution in the form (16) is the existence of the weak ergodicity in a system. Mixing the system states at big times will lead to the distributions (16).

As we note in Conclusion, the structure factor $\omega(E, \Gamma)$ has a meaning of the joint probability density of values $E, \Gamma$. For the distribution (16), the function $\omega(E, \Gamma)$ from $(4),(6),(8)$ takes on the form:

$$
\omega(E, \Gamma=y)=\omega(E) \Gamma_{0}^{-1} \exp \left\{-\frac{y}{\Gamma_{0}}\right\} .
$$

Substituting into the partition function (8) yields

$$
Z(\beta, \gamma)=Z(\beta)\left(1+\gamma \Gamma_{0}\right)^{-1},
$$

where $Z(\beta)=\int \omega(E=x) \exp \{-\beta x\} d x$ is the Gibbs partition function.

We have from (9) and (18) when $\Gamma_{\gamma}=-\partial \ln Z(\beta, \gamma) / \partial \gamma$, $\Gamma_{0}(V)=\left.\Gamma_{\gamma}(V)\right|_{\gamma=0}$,

$$
\Gamma_{\gamma}=\frac{\Gamma_{0}}{1+\gamma \Gamma_{0}}, \quad \gamma=\frac{1}{\Gamma_{\gamma}}-\frac{1}{\Gamma_{0}},
$$

that is, $\gamma$ is the difference between the inverse lifetimes of the open system $1 / \Gamma_{\gamma}$ and the system without external influences $1 / \Gamma_{0}$ which can degenerate only because of its internal fluctuations. The value $\gamma$ is thus responsible for describing the interaction with the environment, and its existence is the consequence of the open character of a system. When defining $\gamma$, one should take into account all factors which contribute to the interaction between the system and the environment. If one denotes in (19) $x=\gamma \Gamma_{0}$, then $x=$ $x_{1}+x_{2}+\cdots+x_{n}$, where the value $x_{i}$ is determined by the flux labelled by the index $i$. Below we shall treat only the case of one flux without conservative external fields and velocity gradients and the equation for the specific energy $u$ has the form $\rho d u / d t+\vec{\nabla} \vec{J}_{q}=0, E=\int_{V} \rho u d V, \rho=M / V$ is the mass density, and $\overrightarrow{J_{q}}=\overrightarrow{J_{u}}=\vec{q}$ is the heat flux. We consider the case of the constant mass density.

Let us introduce the nonequilibrium entropy corresponding to the distribution (7) in the analogy to [17] by the relation

$$
\frac{S}{k_{B}}=-\langle\ln \rho(z ; E, \Gamma)\rangle=\beta\langle E\rangle+\gamma\langle\Gamma\rangle+\ln Z(\beta, \gamma) .
$$

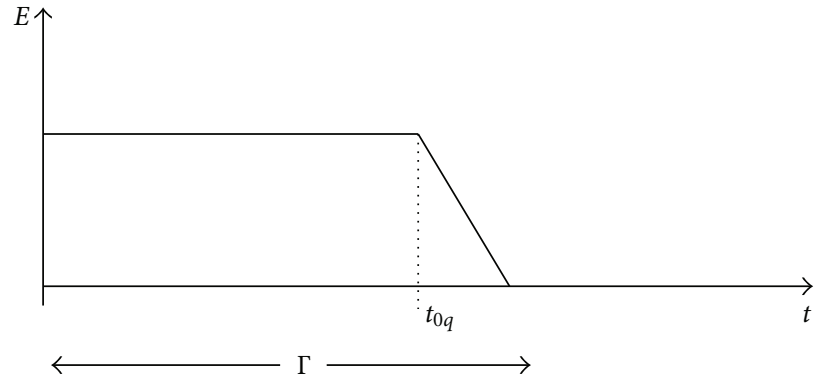

Figure 2: Geokinetic plateau model.

For the case of several potential wells (in contrast to Figure 1) with the same minima positions (which means the case of a system with several ergodic states), one can expect the appearance of the Erlang-type distributions instead of the exponential one (16) [23]. More precise approximations can also be used.

\section{One-Phase Systems (Systems Possessing One Class of the Stable States), Heat Conductivity, Mass Transfer, and Chemical Reactions}

Substituting into (20) the expressions (18) and (19), we have

$$
\begin{gathered}
\frac{S}{k_{B}}=\frac{S_{\beta}}{k_{B}}+\frac{x}{(1+x)}-\ln (1+x), \\
\frac{S_{\beta}}{k_{B}}=\beta\langle E\rangle+\ln Z_{\beta}, \quad x=\gamma \Gamma_{0} .
\end{gathered}
$$

From (20) treating $E$ and $\Gamma$ as variables,

$$
d S=k_{B} \beta d E+k_{B} \gamma d \Gamma \text {. }
$$

From (19), $d \Gamma=-\Gamma^{2} d \gamma+\left(\Gamma / \Gamma_{0}\right)^{2} d \Gamma_{0}$. Substituting into (22), we find from the Maxwell relations that $\partial \Gamma_{0} / \partial E$ and $\partial \Gamma_{0} / \partial R$ are proportional to $\partial \Gamma_{0} / \partial \vec{q}=0$ (since $\Gamma_{0}$ by definition corresponds to the state with $\vec{q}=0$ and $\Gamma_{0}$ independent on $\vec{q})$. Thats why $d \Gamma_{0}=0$ and

$$
d S=k_{B} \beta d E-\frac{k_{B} x d x}{(1+x)^{2}},
$$

which coincides with the differential of (21).

Now we determine the explicit form for $y$ and $x$. Considering the simplest model, for example, that of the geokinetic plateau [7], Figure 2, which features a system in the stationary state, which degenerates during a short-time interval $t_{0 q}$, changing from a stationary state to zero state, we can obtain for the heat conductivity in this case

$$
\begin{gathered}
x_{q}=\gamma_{q} \Gamma_{0} \approx \frac{t_{0 q} S_{a} q}{E}=\frac{t_{0 q} q}{\rho u R}=t_{0 q} y_{q}, \\
y_{q}=\frac{q}{\rho u R}, \quad E \approx \rho u V,
\end{gathered}
$$

where $q= \pm|\vec{q}|=(\vec{q} \vec{q})^{1 / 2}, u=E / \rho V$, energy density in a system, $R$ its linear size, $V$ its volume $\sim R^{3}$, and 


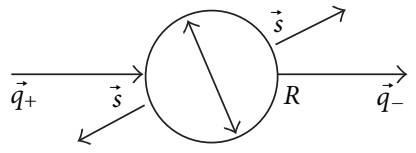

Figure 3: The volume element of the size $R$, incoming $\vec{q}_{+}$, and outcoming $\vec{q}$ - fluxes, normal to the surface element $\vec{s}$.

the surface $S_{a} \sim R^{2} ; q_{+}=\left(\vec{q}_{+} \vec{s}\right)=q=(\vec{q} \vec{q})^{1 / 2}$, where $q_{-}$ and $q_{+}$are projections of the outcoming and incoming heat flux density vectors on the surface normal vector $\vec{s}$ (ordered chronologically, Figure 3 ); the signs of $q_{-}$and $q_{+}$depend on whether we are heating or cooling the system. Then $q_{+}=$ $-q_{-}-R \rho \partial u / \partial t$, in the stationary state $q_{+}=-q_{-}$.

Outcoming flux $\vec{q}_{-}$and incoming flux $\vec{q}_{+}$differ in their times: we consider $\vec{q}_{+}$to be the first flux. In (24) we suppose the finite velocity of the heat propagation in the system. Thus the linear dimensions enter to the characteristics of the "point" system. For continuous systems $R=L$, where $L$ is the size of the "point" of the continuous medium in the continuous description $[20,22]$. In the frame of the kinetic theory $L=l_{\mathrm{ph}}=l_{1}\left(\varepsilon_{1}\right)^{1 / 2} \ll l_{1}, \varepsilon_{1}=n r_{0}^{3}$, where $n$ is the density, $r_{0}$ is the diameter of the atom, $l_{1}$ is the free path length. In the gasodynamic description $L=l_{p h}^{G} \sim L^{G} / N^{1 / 5}$, where $L^{G}$ is the size of the system, $N$ is the particle number. One should take into account the source density $\sigma$ when describing the reaction systems. For example, in the nuclear reactors one must take into account the terms responsible for the fuel sources. But even in the equations for the internal energy one should account for the sources if external fields and/or inner heterogeneities are present [3]. Let the system be open with respect to the quantity $A=\int_{V} \rho a d V$, for which the balance equation $\partial(\rho a) / \partial t+\vec{\nabla} \overrightarrow{J_{a}^{0}}=\sigma_{a} ; \overrightarrow{J_{a}^{0}}=\rho_{a} \overrightarrow{v_{a}}[3]$ holds ( $\vec{v}$ is the velocity, with $\rho=M / V$ being the mass density); $\operatorname{div} \overrightarrow{J_{a}^{0}} \approx\left(J_{a-}^{0}+J_{a+}^{0}\right) / R$, where $R$ is the "size" of the point in the continuous description [22]. Then the value $J_{a+}^{0}-R \sigma_{a}=$ $-J_{a-}^{0}-R \partial(\rho a) / \partial t$ is proportional to $\gamma$ from (19). And the value $y_{a}=\left(J_{a+}^{0}-R \sigma_{a}\right) / R a$, multiplied by the time parameter $t_{0 a}$ (from (28)), coincides with $x=\gamma \Gamma_{0}$. The equation for the specific energy $u$ has the form $\rho d u / d t+\vec{\nabla} \overrightarrow{J_{q}}=0 ; E=$ $\int_{V} \rho u d V ; \overrightarrow{J_{q}}=\overrightarrow{J_{u}}=\vec{q}$ is the heat flux. We consider the case of the constant mass density.

The same result (24) can be obtained if we compare the entropy of macroobservables $E$ and $\vec{q}$ of EIT [4] with the entropy of macroobservables $E$ and $\Gamma$. [4]:

For this purpose, we will recall the expressions of the EIT

$$
S=S_{\beta}-\frac{\int_{V} \rho \alpha_{q}(\vec{q} \vec{q}) d \vec{r}}{2}, \quad \alpha_{q}=\frac{\tau_{q}}{\rho \lambda T^{2}}
$$

( $\vec{q}$ and $\tau_{q}$ are values from (1)). Comparing with (21) at small $\vec{q}: S=S_{\beta}-k_{B} x^{2} / 2, k_{B} x_{q}^{2}=\int_{V} \rho \alpha_{q}(\vec{q} \vec{q}) d \vec{r}$,

$$
x= \pm\left(\frac{\int_{V} \rho \alpha_{q}(\vec{q} \vec{q}) d \vec{r}}{k_{B}}\right)^{1 / 2}=\gamma \Gamma_{0} .
$$

The sign "-" in (26) is chosen if the incoming flux is directed towards the system (the system is being heated). The derivative $\partial x /\left.\partial \vec{q}\right|_{E, R}$ is written as $\int_{V}\left[\delta_{x} /\left.\delta \vec{q}(\vec{r})\right|_{E, R}\right] d \vec{r}=$ $\int_{V} \rho \alpha_{q} \vec{q} d \vec{r} / k_{B} x$, where $R$ is the linear size of the system. The expressions for $\partial x /\left.\partial E\right|_{q, R}, \partial x /\left.\partial R\right|_{E, q}$ can be obtained in the same fashion. Then from (23)

$$
\begin{aligned}
d S= & \left\{k_{B} \beta-\frac{\int_{V}\left[\partial\left(\rho \alpha_{q}\right) / \partial u\right](\vec{q} \vec{q}) d \vec{r}}{\rho V 2(1+x)^{2}}\right\} d E \\
& -\frac{\int \rho \alpha_{q} \vec{q} d \vec{r} d \vec{q}}{(1+x)^{2}}-\frac{2 x^{2} k_{B} d R}{R(1+x)^{2}} .
\end{aligned}
$$

If the fluxes $q$ depend only weakly on the spatial variables, then (26) is cast as

$$
\begin{gathered}
x_{q} \approx \pm\left(\frac{\int_{V} \rho \alpha_{q} d \vec{r}}{k_{B}}\right)^{1 / 2}(\vec{q} \vec{q})^{1 / 2} \\
\approx \frac{t_{0 q} S_{a} q}{E}=\frac{t_{0 q} q}{\rho u R}=t_{0 q} y_{q}, \\
t_{0 q} \approx E \frac{\left(\int_{V} \rho \alpha_{q} d \vec{r} / k_{B}\right)^{1 / 2}}{S_{a}}, \quad y_{q}=\frac{q}{\rho u R}, \quad E \approx \rho u V,
\end{gathered}
$$

which coincides with (24).

From (23) we have

$$
\begin{aligned}
d S= & k_{B}\left[\beta+\frac{x^{2}}{E(1+x)^{2}}\right] d E \\
& -\left[\frac{k_{B} x^{2}}{q(1+x)^{2}}\right] d q-\left[\frac{k_{B} 2 x^{2}}{R(1+x)^{2}}\right] d R .
\end{aligned}
$$

The behaviour of a system depends on its finite size, since we consider the systems with finite lifetime and finite volumes without performing the thermodynamic limit transition.

Consider now the stability of the thermodynamic system. To ensure its stability, the condition $\delta^{2} S \leq 0$ should satisfy. We have

$$
\begin{gathered}
\frac{k_{B}{ }^{-1} \partial^{2} S}{\left.\partial E^{2}\right|_{q, R}}=\frac{\partial \beta}{\partial E}-\frac{x^{2}(3+x)}{E^{2}(1+x)^{3}}, \quad \frac{k_{B}{ }^{-1} \partial^{2} S}{\left.\partial q^{2}\right|_{E, R}}=\frac{x^{2}(x-1)}{q^{2}(1+x)^{3}}, \\
\frac{k_{B}{ }^{-1} \partial^{2} S}{\left.\partial R^{2}\right|_{E, q}}=\frac{2 x^{2}(x-3)}{R^{2}(1+x)^{3}}, \quad \frac{k_{B}{ }^{-1} \partial^{2} S}{\partial E \partial q}=\frac{2 x^{2}}{q E(1+x)^{3}}, \\
\frac{k_{B}{ }^{-1} \partial^{2} S}{\partial q \partial R}=\frac{-4 x^{2}}{q R(1+x)^{3}}, \quad \frac{k_{B}{ }^{-1} \partial^{2} S}{\partial E \partial R}=\frac{4 x^{2}}{E R(1+x)^{3}}, \\
\frac{\partial \beta}{\partial E}=\frac{-1}{k_{B} T^{2} c},
\end{gathered}
$$

$c=\partial E / \partial T$ is the heat capacity. The nonequilibrium heat capacity $c_{N}=\partial E / \partial \theta$ is likewise defined. If $R=$ const (variables are $E$ and $q$ ), the condition $\delta^{2} S \leq 0$ holds for $\partial S^{2} /\left.\partial E^{2}\right|_{q, R} \leq 0, \partial^{2} S /\left.\partial q^{2}\right|_{E, R} \leq 0$. These conditions 
satisfy when $-1 / T^{2} c-k_{B} x^{2}(3+x) / E^{2}(1+x)^{2} \leq 0$ and $k_{B} x^{2}(x-1) / q^{2}(1+x)^{3} \leq 0$. One more condition satisfies if $\left(\partial S^{2} /\left.\partial E^{2}\right|_{q, R}\right)\left(\partial^{2} S /\left.\partial q^{2}\right|_{E, R}\right)-\left(\partial^{2} S / \partial E \partial q\right)^{2} \geq 0$. Hence

$$
|x| \leq\left(1+\frac{k_{B} T^{2} c}{E^{2}}\right)^{-1 / 2} .
$$

It is seen from (31) that at $q<0, x<0, c>0,|x|<1$ that is the denominator in (19), (29) does not equal zero in the domain of the thermodynamic stability and the expression for $\Gamma$ converge. The same relations are written for $E=$ const in the variables $q, R$ and for $q=$ const in terms of $E, R$.

Determining in (29) $d S / d t$, we find the entropy balance equation $(d S / d t) / V=-\vec{\nabla} \overrightarrow{j_{S}}+\sigma_{S}, \quad S=\int_{V} \rho s d \vec{r}$, where for $\rho=$ const, $\overrightarrow{j_{S}}=\theta^{-1} \vec{q}$,

$$
\begin{aligned}
\sigma_{S}= & \vec{q} \vec{\nabla} \theta^{-1}-\frac{k_{B} x^{2}(d q / d t)}{q V(1+x)^{2}} \\
& +\frac{k_{B}(d R / d t)\left[3 u \beta \rho+x^{2} V^{-1}(1+x)^{-2}\right]}{R} .
\end{aligned}
$$

Comparing entropy production $\sigma_{S}$ and $\sigma_{S}=\vec{q} \vec{q} / \lambda T^{2}[3]$, we shall find

$$
\begin{aligned}
\lambda T^{2} k_{B} x^{2}\left(\frac{d \vec{q}}{d t}\right) q^{-2} V^{-1}(1+x)^{-2}+\vec{q} \\
=\lambda T^{2} \nabla \theta^{-1}+\left(\frac{d R}{d t}\right) \frac{\lambda T^{2} k_{B}\left[3 u \beta \rho+x^{2} V^{-1}(1+x)^{-2}\right]}{\vec{q} R} .
\end{aligned}
$$

The inverse nonequilibrium temperature

$$
\begin{aligned}
\theta^{-1} & =\left.\frac{\partial S}{\partial E}\right|_{q, R}=k_{B}\left[\beta+\frac{x^{2}}{E(1+x)^{2}}\right] \\
& =\frac{1}{T}+\frac{q^{2} \alpha_{q}}{u\left[1+q\left(\rho \alpha_{q} V / k_{B}\right)^{1 / 2}\right]^{2}}
\end{aligned}
$$

coincides with the value $1 / \theta$ of the extended thermodynamics [4] at small $q$ (when $(1+x)^{-2} \approx 1$ ). The value entropy production $\sigma_{S}$ and the expression (33) when substituting therein (28) take on the form

$$
\begin{aligned}
& \sigma_{S}= \vec{q} \vec{\nabla} \theta^{-1}-\rho q \alpha_{q} \frac{(d q / d t)}{(1+x)^{2}} \\
&+\frac{(d R / d t) \rho\left[3 u T^{-1}+q^{2} \alpha_{q}(1+x)^{-2}\right]}{R}, \\
&\left(\frac{d \vec{q}}{d t}\right) \tau_{q}(1+x)^{-2}+\vec{q} \\
&=\lambda T^{2} \vec{\nabla} \theta^{-1}+\frac{(d R / d t)\left[3 u \lambda T \rho / \vec{q}+\vec{q} \tau_{q}(1+x)^{-2}\right]}{R},
\end{aligned}
$$

and coincide with the corresponding expressions of EIT and Maxwell-Cattaneo equation (1) at $d R / d t=0$ and small $q$. The same can be derived from (27).

Above we considered the lifetime as a quantity related to the heat in a system. Since the heat transfer in a body is accompanied by the processes of deformation of a continuous medium, the energy dissipation is conditioned not only by the heat transfer, but by the internal friction of a system which is represented by the dissipative part of the stress tensor; thus the full expression for $x$ must have (similar to $[24])$ the form

$$
x= \pm\left(\int_{V} \frac{d \vec{r}}{k_{B}} \rho\left[\frac{\tau_{q}}{\rho \lambda T^{2}}(\vec{q} \vec{q})+\left(\sigma^{v}: \sigma^{v}\right) \frac{\tau_{v}}{\rho \mu T}\right]\right)^{1 / 2},
$$

where $\sigma^{v}$ is the viscous stress tensor, $\tau_{v}$ is the correlation time of viscous stresses, and $\mu$ is the shear viscosity. Correspondingly, the expressions for $\overrightarrow{j_{S}}, \sigma_{S}, \ldots$ acquire more cumbersome form. Similar expressions can be written down for the density changes (if one considers the lifetime for the full mass), for velocity and for other factors contributing to the expression for $\sigma_{u}$ from the equation for the specific energy density [3], and so forth.

In the examples considered above, one took for $\beta E$ the values $\int d r \beta(r, t) \hat{u}(r)$, where $\hat{u}(r)$ is the dynamical variable of the energy density, $\beta(r, t)=1 / k_{B} T(r, t)$; if we consider the processes with variable mass, we should take as $\beta E$ the quantities $\int d r \beta(r, t)\left[\hat{u}(r)-\sum_{k} \mu_{k}(r, t) \hat{n}_{k}(r)\right]$, where $\hat{n}_{k}(r)$ is dynamical variable of the particle density for the particles of $k$-th kind, $\mu_{k}(r, t)$ is the chemical potential of the $k$-th particles. Taking into account the chemical reactions like $\sum_{j=1}^{r} v_{j}^{+} X_{j} \stackrel{k_{+}}{\longleftrightarrow} \sum_{j=1}^{r} v_{j}^{-} X_{j}$, we get

$$
x_{\rho}= \pm \sum_{k=1}^{r}\left(\int_{V} \frac{d \vec{r}}{k_{B}}\left[\frac{\tau_{k \text { diff }}}{L_{k} V}\left(\vec{j}_{k} \vec{j}_{k}\right)+\left(\omega_{k} \omega_{k}\right) \frac{\tau_{k c h e m}}{L_{k \text { chem }} V T}\right]\right)^{1 / 2},
$$

where $x_{k}=t_{0 k}\left(J_{k}-\omega_{k} R\right) / R c_{k}, t_{0 k}=t_{0 k \text { diff }}=c_{k} R\left(\tau_{\rho \text { diff }} / k L_{k}\right)^{1 / 2}$, where $\tau_{\rho \text { diff }}$ is the correlation time for the fluxes $\vec{J}_{k}, J_{k}$ is the normal projection of $\overrightarrow{J_{k}}$ (the incoming flux into the system of the size $R$ ) to the surface, $L_{k}=D_{k} T /\left(\partial \mu_{k} / \partial c_{k}\right)_{T, P}, \mu_{k}$ is the chemical potential $\left(=\mu_{0 k}\right.$ without perturbation), and $L_{k}$ is Onsager coefficient from $\overrightarrow{J_{i}}=-L_{i} \vec{\nabla}\left(\mu_{i} / T\right)_{T, P}$. We consider a multicomponent mixture with mass densities of the components $\rho_{k}$ and concentrations $c_{k}=\rho_{k} / \rho, k=1, \ldots, r$; $\sum_{k=1}^{r} \rho_{k}=\rho, c_{n}=N_{n} / V$, where $r$ is the total number of particle types involved into reactions, $N_{n}$ is the number of particles of the $n$-th type. The balance equation for $c_{j}$ has the form $\partial c_{j} / \partial t=-\vec{\nabla} \overrightarrow{J_{j}}+\omega_{j}$, where $\overrightarrow{J_{j}}=-D_{j} \vec{\nabla} c_{j}$ is the diffusive flux of the $j$-th component, $D_{j}$-its diffusion coefficient, $\omega_{j}=k+\prod_{n=1}^{r} c_{n}^{\nu_{n}^{+}}-k_{-} \prod_{n=1}^{r} c_{n}^{v_{n}^{-}}$the rate of the $j$-th reaction per unit volume, $k_{+}$and $k_{-}$are reaction constants, $v_{n}=v_{n}^{-}-v_{n}^{+}$are stechiometric coefficients [3,25]. Besides the value $t_{0 k \text { diff, }}$ one can also introduce the time $t_{0 \mathrm{kchem}}=\left(\alpha_{\rho k \mathrm{chem}} V / k\right)^{1 / 2}, \alpha_{\rho \mathrm{kchem}}=\tau_{\text {kchem }} /\left(V T L_{k \mathrm{chev}}\right)$, $L_{k \text { chem }}=c_{k} \omega_{+}^{\mathrm{eq}} / k T, \omega_{+}^{\mathrm{eq}}=k_{+} \prod_{j=1}^{r}\left(c_{j}^{\mathrm{eq}}\right)^{v_{j}^{+}}$(such choice 
of kinetic coefficients for chemical reactions where $\omega=$ $L A\left(A=-\sum_{n=1}^{r} v_{n} \mu_{n}=-k T \ln \prod_{j=1}^{r}\left(c_{j} / c_{j}^{\text {eq }}\right)^{v_{j}}\right.$ is chemical affinity) is performed, e.g., in [25]), and $\tau_{k \text { chem }}$ is the correlation time of the fluxes $\omega_{k}$ caused by chemical reactions. Since $t_{0 k c h e m}$ and $t_{0 k \text { diff }}$ enter the same balance equation for $c_{k}, t_{0 k \mathrm{diff}}=t_{0 \mathrm{kchem}}$. From this, we get the relation between $\tau_{k c h e m}$ and $\tau_{k \text { diff: }} \tau_{k c h e m} /\left(T L_{k \text { chem }}\right)=R^{2} c_{k}^{2} \tau_{\rho \text { diff }} / L_{k}$. As thermodynamic quantities, let us take $c_{k}, J_{k}, m_{k}, m_{k}=$ $\sum_{j=1}^{r} \gamma_{k j} \omega_{j}, \omega_{j}=\left(\partial_{j} \rho_{k} / \partial t\right) / \gamma_{k j}$ in the $j$-th chemical reaction [3], $\sum_{k=1}^{K} m_{k}=0$. Since $\partial c_{k} / \partial \rho_{k}=\left(1-c_{k}\right) / \rho, \partial x_{k} / \partial c_{k \mid J_{k}, m_{k}}=$ $-x_{k}\left(\partial \rho_{k} / \partial c_{k}\right) / \rho_{k}=x_{k} / c_{k}\left(c_{k}-1\right), \partial x_{k} / \partial J_{k \mid c_{k}, m_{k}}=x_{k} m_{k} R /$ $J_{k}\left(J_{k}-R m_{k}\right), \partial x_{k} / \partial m_{k \mid c_{k}, J_{k}}=-R x_{k} /\left(J_{k}-R m_{k}\right)$. Substituting these expressions into (3), we get

$$
\left(k_{B}\right)^{-1} d S=\left(k_{B}\right)^{-1} d S_{\beta}-(\gamma \Gamma)^{2} \sum_{k=1}^{r} \frac{\gamma_{k}[\mathfrak{A}]}{\gamma},
$$

where $\mathfrak{A}$ denotes $d c_{k} / c_{k}\left(c_{k}-1\right)+R m_{k} d J_{k} / J_{k}\left(J_{k}-R m_{k}\right)-$ $R d m_{k} /\left(J_{k}-R m_{k}\right)$.

If we substitute $d c_{k} / d_{t}$ from the balance equation and use the transformation $\mu \vec{\nabla} J_{k} / k_{B} T=\vec{\nabla}\left(\mu J_{k} / k_{B} T\right)-J_{k} \vec{\nabla}\left(\mu / k_{B} T\right)$, we get the entropy balance equation with the entropy flux $\overrightarrow{j_{S}}=-\sum_{k} \overrightarrow{J_{k}} \mu_{k} / T, \mu_{k} / k_{B} T=\mu_{0 k} / k_{B} T+(\gamma \Gamma)^{2} \gamma_{k} / \gamma c_{k}\left(c_{k}-1\right)$, and entropy production $\sigma_{S}=\sum_{k=1}^{r}\left\{-\overrightarrow{J_{k}} \vec{\nabla}\left(\mu_{k} / T\right)-\mu_{k} m_{k} / T-\right.$ $\rho \gamma \Gamma^{2} k_{B} \gamma_{k}\left[R m_{k}\left(d J_{k} / d t\right) / J_{k}\left(J_{k}-R m_{k}\right)-R\left(d m_{k} / d t\right) /\left(J_{k}-\right.\right.$ $\left.\left.\left.R m_{k}\right)\right]\right\}$. Setting this value $\sigma_{S}$ equal to $\sigma_{S}=\sum_{k=1}^{r} J_{k}^{2} / L_{k k}+$ $\sum_{r=1}^{s} \sum_{l=1}^{s} R_{r l} \omega_{r} \omega_{l} / T$, where $R_{r l}$ are resistivity matrices [3] from the expression $A_{r}=\sum_{l=1}^{s} R_{r l} \omega_{l}$, $(s$, number of chemical reactions in a system) we get the general expression relating $d J_{k} / d t, d m_{k} / d t$ with $J_{k}, m_{k}$. Further, considering $m_{k} R \ll$ $J_{k}$ (almost pure diffusion), one can get an equation for $J_{k}$, similar to (9) for $q$. Vice versa, when $J_{k} \ll m_{k} R$ (that is, chemical reactions only), one can get an equation for $m_{k}$ and $\omega_{j}$. The stability conditions are written similarly to the heat conductivity equation.

\section{Stationary Nonequilibrium States}

We suppose that the expressions (20), (21) which are results of the present paper are satisfied for arbitrary values of fluxes. Thats why they are believed to be capable of describing the stationary nonequilibrium states far from equilibrium in a more accurate fashion than EIT does. Linear deviations should thus be calculated starting from some reference states determined in this case by the stationary values of fluxes. For the distribution (16), one should generate the expansion round about the value $x_{0}=x_{\mathrm{st}}=q_{0} R^{2} t_{0} / E_{0}, E_{0}=E_{\mathrm{st}}$, $q_{0}=q_{\mathrm{st}}, \gamma_{0}=\gamma_{\mathrm{st}}=\varepsilon q_{0} R^{2} / E_{0}$.

The expression for the entropy thus has a form

$$
\begin{aligned}
\frac{S}{k_{B}}= & \frac{S_{\beta}}{k_{B}}+\frac{x_{0}}{\left(1+x_{0}\right)}-\ln \left(1+x_{0}\right) \\
& -\frac{\left(x-x_{0}\right) x_{0}}{\left(1+x_{0}\right)^{2}}+\frac{\left(x-x_{0}\right)^{2}\left(x_{0}-1\right)}{2\left(1+x_{0}\right)^{3}}+\cdots .
\end{aligned}
$$

If one introduces the lifetime in a stationary nonequilibrium state $\Gamma_{1}\left(\gamma_{0}\right)=\Gamma_{0} /\left(1+\gamma_{0} \Gamma_{0}\right), x_{1}=\gamma_{0} \Gamma_{1}\left(\gamma_{0}\right)=x_{0} /\left(1+x_{0}\right)$, then the latter expression is cast as

$$
\begin{aligned}
\frac{S}{k_{B}}= & \frac{S_{\beta}}{k_{B}}+x_{1}+\ln \left(1-x_{1}\right)-\left(x-x_{0}\right) x_{1}\left(1-x_{1}\right) \\
& +\frac{\left(x-x_{0}\right)^{2}\left(2 x_{1}-1\right)\left(1-x_{1}\right)^{2}}{2}+\cdots .
\end{aligned}
$$

\section{Conclusion}

The assumption about the physical systems living for a finite period of time which was the starting point of the exposed here theory allows one to get the mesoscopic theory of the stationary nonequilibrium states at any deviation from the equilibrium. For the method applied, it is essential to have the relation of $\Gamma$ as a slave process to the master process $E(t)$. But the concept of the lifetime has a more profound physical sense to cast in one fashion the Newtonian approach to the absolute time and the concepts of time-generating matter. The lifetime parameters is a compromising concept uniting in itself the properties of ordinary dynamical values like energy and the particle number are and the coordinate variables like the time variable is. Mathematically introducing lifetime means yielding an additional information on the stochastic process besides its stationary distribution leaning upon the stationary properties of the slave process. Distribution of the form (3)-(9) studied in [26, 27]. Statistical justification for introducing such distributions is given in [28].

Let us underline the principal features of the suggested approach.

(1) We introduce a novel variable $\Gamma$ which can be used to derive additional information about a system in the stationary nonequilibrium state. We suppose that $\Gamma$ is a measurable quantity at macroscopic level, thus values like entropy which are related to the order parameter (principal macroscopic variable) can be defined. At the mesoscopic level, the variable $\Gamma$ is introduced as a variable with operational characteristics of a random process slave with respect to the process describing the order parameter.

(2) We suppose that thermodynamic forces $\gamma$ related to the novel variable can be defined. One can introduce the "equations of state" $\beta(\langle E\rangle\langle\Gamma\rangle), \gamma(\langle E\rangle,\langle\Gamma\rangle)$. Thus we introduce the mapping (at least approximate) of the external restrictions on the point in the plane $\beta, \gamma$.

(3) We suppose that a "refined" structure factor $\omega(E, \Gamma)$ can be introduced which satisfies the condition $\int \omega(E, \Gamma=$ $y) d y=\omega(E)$ (ordinary structure factor). This function (like $\omega(E)$ ) is the internal (inherent) property of a system. At the mesoscopic level, we can ascribe to this function some inherent to the system (at given restrictions $\left(\beta_{0}, \gamma_{0}\right)$ ) random process. The structure factor has the meaning of the joint probability density for the values $E, \Gamma$ understood as the stationary distribution of this process. Provided the "reper" random process for the point $\left(\beta_{0}, \gamma_{0}\right)$, one can derive therefrom the shape of the structure function. If we model the dependence of the system potential of the order parameter by some potential well, the lifetime distribution within 
one busy period and probabilities $\omega(E, \Gamma)$ can be viewed as distributions of the transition times between the subset of the phase space (possibly of the fractal character) corresponding to the potential well, and the subset corresponding to the domain between the "zero" and the "hill" of the potential wherefrom the system will roll down to the zero state (i.e., from the domain $y>b$ towards $y<b$, Figure 1). To determine the explicit form of $\Gamma\left(\right.$ at $\left.\left(\beta_{0}, \gamma_{0}\right)\right)$, the algorithm of the asymptotic phase coarsening of complex system is used.

(4) It is supposed that at least for certain classes of influences the resulting distribution has the form (4), (7), that is, the change of the principal random process belongs to some class of the invariance leading to this distribution, which explains how one can pass from the process in the reper point $\left(\beta_{0}, \gamma_{0}\right)$ (e.g., in equilibrium when $\gamma=0$ and $\left.\beta=1 / k_{B} \mathrm{~T}\right)$ to a system in an arbitrary nonequilibrium stationary. The thermodynamic forces should be chosen so that the distribution leads to new (measurable) values of $(\langle E\rangle,\langle\Gamma\rangle)$.

(5) The values $\gamma$ and $x$ are determined from the comparison with EIT.

If one integrates (4) and (7) over $\Gamma$, one gets the distribution $P(E)$ depending on $E$ as well as on $\beta$ and $\gamma$ as parameters:

$$
P(E)=\int P(E, \Gamma=y) d y=\frac{\exp \left\{-\beta E-\gamma \tau_{\gamma}\right\} \omega(E)}{Z(\beta, \gamma)},
$$

where $\gamma \tau_{\gamma}=-\ln L(\gamma, E)$ and $L(\gamma, E)=\int e^{-\gamma y} \omega(E, \Gamma=$ $y) d y / \omega(E)$. Averaging the expression for $\ln L(\gamma, E)$, we shall get for $\tau=-\langle\ln L(\gamma, E)\rangle / \gamma$,

$$
\begin{aligned}
\langle\ln L(\gamma, E)\rangle & =\frac{\int \exp \{-\beta x-\gamma y\} \omega(x, y) \ln L(\gamma, x) d x d y}{Z(\beta, \gamma)} \\
& =\frac{\int \exp \{-\beta x\} \omega(x) L(\gamma, x) \ln L(\gamma, x) d x}{Z(\beta, \gamma)} .
\end{aligned}
$$

Let us introduce the value $\tau_{0}=\langle\Gamma\rangle-\tau=$ $\int \exp \{-\beta x\} \omega(x)[L \ln L-\gamma \partial L / \partial \gamma] d x / \gamma Z(\beta, \gamma)$, which vanishes at $\gamma=0$. The value $S-S_{\beta}=\Delta S=\gamma \tau_{0}(<0)$ is the loss of information when passing from the distribution (7), (4) to (41).

Essential assumption is that the values $\Gamma_{0}$ and $\ln L(\gamma, E)$ do not depend upon the initial value of $E=x$, that is, the value $\Gamma_{0}$ can be understood as an average on $\langle E\rangle$ rather than of dynamical random variable $E$. This fact is the direct consequence of the Markoff chain subject to the unperturbed process being equally ergodic and possessing a single (for the distribution (16)) class of the ergodic states (Appendix B). Then the entropy $S_{\langle E\rangle}=-\langle\ln \rho(z ; E)\rangle=\beta\langle E\rangle+\gamma \tau+\ln Z(\beta, \gamma)$ as an average of the distribution (42) coincides with $S_{\beta}$; that is, the correspondence principle holds: the distribution (42) coincides with the Gibbs one. In Section 2 (expressions (3)(9)), we performed the splitting of the phase space into the cells $(E, \Gamma)$ giving up the equiprobable distribution of points in $\omega(E)$. But although we are now ascribing different weights to different points, collecting them together (summation over $\Gamma$ ) yields the Gibbs distribution.
The lifetime (or escape time if one refers to the terminology of [10-12]) is described by smooth differentiable distributions (16)-(17) rather than by singular measures of the fractal repeller object (like it was done in [10-12]). This transition is possible because of use of the complex system coarse-graining algorithm [23]. But in the very general case, the lifetime in the phase space should be of pronounced fractal character. The distribution density (16) can be obtained out of the maximum-entropy principle at given $\Gamma_{0}$ value (when $\Gamma_{0}$ does not depend on the random value $E$ ). The entropy (20) behaves for $\gamma<0$ and $\gamma>0$ in a different fashion, with this property corresponding to the irreversibility since different signs of $\gamma$ mean different signs of the fluxes $q$. The Gibbs theorem and H-theorem [22] hold: the entropy maximum indeed is attained at zero deviations from the equilibrium $\gamma=0$. The irreversibility in this approach appears as a consequence of the lifetime finiteness hypothesis.

If one compares the exposed thermodynamics with EIT, following differences can be outlined.

(1) Different expressions for the nonequilibrium temperature, entropy $S$, entropy production (which yield the EIT expressions as a particular case for small $\vec{q}$ ).

(2) A new variable of the system size is introduced which should play certain part in the nonequilibrium case. For the continuous description, this might be the size of the "continuous medium point" [22].

(3) Explicit expressions for the lifetime $\Gamma$ and its thermodynamic conjugate $\gamma$ are obtained.

\section{Appendices}

\section{A. Markovian Stochastic Model}

Let the process $y(t)$ be Markovian. For the kinetic coefficients

$$
\begin{gathered}
K_{\alpha_{1} \ldots \alpha_{m}}(y)=\lim _{\tau \rightarrow 0}\left[\tau^{-1}\left\langle\Delta y_{\alpha_{1}} \ldots \Delta y_{\alpha_{m}}\right\rangle_{y}\right], \\
\Delta y=y(t+\tau)-y(t),
\end{gathered}
$$

the potential [25] is written

$$
V(\theta, y) \equiv \sum_{m=1}^{\infty} \beta^{m-1}\left(\frac{1}{m !}\right) \sum_{\alpha_{1} \cdots \alpha_{m}} K_{\alpha_{1}} \cdots \alpha_{m}(y) \theta_{\alpha_{1}} \cdots \theta_{\alpha_{m}},
$$

where $\beta^{-1}$ is the small parameter; for the equilibrium Gibbs system $\beta=1 / k_{B} T_{\text {eq }}, k_{B}$ being the Boltzmann constant, $T$ is the temperature.

The forward kinetic equation for the distribution density

$$
\frac{\partial p(y, t)}{\partial t}=N_{\partial, y} \Phi\left(-\frac{\partial}{\partial y}, y\right) p(y, t)
$$

where $\Phi$ is the stochastic potential, $\Phi(\theta, y)=\beta V(\theta / \beta, y)$, the operator $N_{\partial, y}$ defines the order of operations (differentiating on y goes the last). 
The Laplace transform gives the equation for $Q(10)$ :

$$
\frac{\partial Q(\exp \{-\theta\}, t)}{\partial t}=N_{\theta, \partial / \partial \theta} \Phi\left(-\theta,-\frac{\partial}{\partial \theta}, t\right) Q(\exp \{-\theta\}, t) .
$$

One can show that the lifetime (2) is governed by the Hermitian conjugate operator and for $L$ (11) the following holds:

$$
N_{x, \partial} \Phi\left(\frac{\partial}{\partial x}, x\right) L(x, s)=s L(x, s)
$$

with the condition $L(0, s)=L(x, 0)=1$. For the nonhomogeneous Markoff systems if $L$ depends on the initial time $t_{0}$, equation (A.5) is generalized

$$
\begin{aligned}
s L_{t_{0}}(x, s)= & \frac{\partial L_{t_{0}}(x, s)}{\partial t_{0}} \\
& +s N_{x, \partial} \Phi\left(\frac{\partial}{\partial x}, x, t \longrightarrow t_{0}-\frac{\partial}{\partial s}\right)\left(\frac{\left[L_{t_{0}}(x, s)-1\right]}{s}\right) .
\end{aligned}
$$

From these expressions, the precise solutions for the Markoff stochastic models of the physical systems can be found. We note bypassing that in (A.2)-(A.6) the coefficients are unperturbed equilibrium values independent on $\beta_{0}, \gamma_{0}$.

\section{B. Algorithms of the Phase Coarsening of the Complex Systems}

One can represent an open thermodynamic system as evolving in a random medium whose mathematical model will be either Markoff renewal processes or semi-Markoff processes. The local characteristics of the system depend on the random medium state. The scheme of the asymptotic phase coarsening present the simplified description of the system evolution in a random medium which can be performed based upon a simple set of heuristic rules [23]. The semi-Markovian process to describe the evolution of a random system is considered in the enlarged time scale.

In our case, the stationary distributions are described by the Gibbs distributions. Absorbing state is the degenerated state of the system with $E=0$, and the degeneracy probability is $P_{0}=1 / Z(\beta)$. The ergodic Markoff chain has the distribution $\rho$. The residence times in the states $x, \theta_{x}$, are given by the distribution functions $G_{x}(t)=P\left\{\theta_{x} \leq t\right\}=$ $P\left\{\theta_{n+1} \leq t \mid \xi_{n}=x\right\}$ ( $\xi_{n}$ are the states of the Markoff chain) and by the average residence times $m(x)=\int_{0}^{\infty}\left(1-G_{x}(t)\right) d t=$ $E \theta_{x}$, which are limited: $0 \leq m(x)<\infty$. Let us assume that a real system has besides the class of the ergodic states the class of the trapping states where the absorbing of the Markoff chain is possible. The phase space of a real system is then $X=X^{0} \cup X^{\prime} \cup X_{0}$, where $X^{0}$ is the class of the ergodic states of the reference enclosed Markoff chain (without taking into account the absorption into $X_{0}$ ), $X_{0}$ are trapping states of an enclosed Markoff chain, and $X^{\prime}$ is the finite set of the states. In [23] it was shown that the coarsened random process is Markovian with two states $X_{0 \text { coars }}$ and $X_{\text {coars }}^{0}$. The residence time in the stable state is distributed exponentially with the parameter

$$
\begin{gathered}
\lambda_{1}=\frac{1}{m}, \quad m=\int_{X^{0}} \frac{\rho(d x) m(x)}{P_{10}}, \\
P_{10}=P\left\{\xi_{n+1, \text { coars }}=X_{0 \text { coars }} \mid \xi_{n, \text { coars }}=X_{\text {coars }}^{0}\right\} .
\end{gathered}
$$

Thus the expression (16) is obtained in which $m=\Gamma_{0}$. The Markoff processes as objects modelling a complex system appear not as a a priori hypothesis but rather as a result of splitting phase space of states and "gluing up" together the states which belong to one and the same class. The algorithm of the phase coarsening shows the natural property of the complex systems: the transitions in a complex system between the classes of states are rather governed by the Markoff property. If one neglects the detailed description of the system evolution (neglecting the transitions within a class), then (at the condition of big enough residence time within a class) the system losses the dependence of the interclass transitions on the behaviour inside of a class, and the residence time in a class is set as a sum of a big (random) number of the random values which are random times of residence in the states; under certain conditions, this residence time in the class can be considered to have an exponential distribution. We have

$$
P\left(\Gamma_{G}>t\right)=\exp \left\{-\frac{t}{m}\right\}, \quad P\left(\Gamma_{x}>t\right) \approx \exp \left\{-\frac{t}{m}\right\},
$$

where $\Gamma_{G}$ is the residence time of a coarsened system before absorption and $\Gamma_{x}$ is the residence time within the class $X^{0}$ with the initial state $x$. The error in the approximation (B.2) is proportional to the degeneracy probability of the Gibbs system $P_{0}=1 / Z_{\beta}$.

\section{References}

[1] S. R. de Groot and P. Mazur, Non-Equilibrium Thermodynamics, Dover, New York, NY, USA, 1954.

[2] I. Prigogine, Inroduction to Non-Equilibrium Thermodynamics, Wiley-Interscience, New York, NY, USA, 1962.

[3] I. Gyarmati, Non-Equilibrium Thermodynamics: Field Theory and Variational Principles, Springer, Berlin, Germany, 1970.

[4] D. Jou, J. Casas-Vazquez, and G. Lebon, Extended Irreversible Thermodynamics, Springer, Berlin, Germany, 1993.

[5] M. A. Leontovich, Introduction to Thermodynamics. Statistical Physics, Nauka, Moscow, Russia, 1985.

[6] W. Feller, An Introduction to Probability Theory and Its Applications, vol. 2, John Wiley \& Sons, New York, NY, USA, 1971.

[7] E. E. Kovalev, A. I. Vichrov, and V. G. Semenov, "Stability of complex systems and technogene risk," Atomnaya Energiya, vol. 83, p. 291, 1997 (Russian).

[8] P. Salamon, A. Nitzan, B. Andresen, and R. S. Berry, "Minimum entropy production and the optimization of heat engines," Physical Review A, vol. 21, no. 6, pp. 2115-2129, 1980.

[9] P. Salamon and R. S. Berry, "Thermodynamic length and dissipated availability," Physical Review Letters, vol. 51, no. 13, pp. 1127-1130, 1983. 
[10] P. Gaspard, "What is the role of chaotic scattering in irreversible processes?" Chaos, vol. 3, no. 4, pp. 427-442, 1993.

[11] P. Gaspard and J. R. Dorfman, "Chaotic scattering theory, thermodynamic formalism, and transport coefficients," Physical Review E, vol. 52, no. 4, pp. 3525-3552, 1995.

[12] P. Gaspard and G. Nicolis, "Transport properties, Lyapunov exponents, and entropy per unit time," Physical Review Letters, vol. 65, no. 14, pp. 1693-1696, 1990.

[13] G. van Kampen, Stochastic Processes in Physics and Chemistry, North-Holland, Amsterdam, The Netherlands, 1981.

[14] E. T. Jaynes, "Information theory and statistical mechanics," Physical Review, vol. 106, no. 4, pp. 620-630, 1957.

[15] E. T. Jaynes, "Information theory and statistical mechanics. II," Physical Review, vol. 108, no. 2, pp. 171-190, 1957.

[16] J. L. del Río-Correa and L. S. García-Colín, "Increase-inentropy law," Physical Review E, vol. 48, no. 5, pp. 819-828, 1991.

[17] D. N. Zubarev, Nonequilibrium Statistical Thermodynamics, Consultants Bureau, New York, NY, USA, 1974.

[18] I. Prigogine, Non-equilibrium Statistical Mechanics, John Wiley \& Sons, New York, NY, USA, 1962.

[19] R. Balescy, Equilibrium and Nonequilibrium Statistical Mechanics, John Wiley \& Sons, New York, NY, USA, 1975.

[20] Yu. L. Klimontovich, Statistical Physics, Harwood Academic Publishers, New York, NY, USA, 1986.

[21] W. Ebeling, "Entropy and information in processes of selforganization: uncertainty and predictability," Physica A, vol. 194, no. 1-4, pp. 563-575, 1993.

[22] Yu. L. Klimontovich, Statistical Theory of Open Systems, Kluwer Academic Publishers, Dordrecht, The Netherlands, 1995.

[23] V. S. Korolyuk and A. F. Turbin, Mathematical Foundations of the State Lumping of Large Systems, Kluwer Academic Publishers, Dordrecht, The Netherlands, 1993.

[24] G. Lebon, "From classical irreversible thermodynamics to extended thermodynamics," Acta Physica Hungarica, vol. 66, no. 1-4, pp. 241-249, 1989.

[25] R. L. Stratonovich, Nonlinear Nonequilibrium Thermodynamics, Springer, Heidelberg, Germany, 1992.

[26] V. V. Ryazanov and S. G. Shpyrko, "First-passage time: a conception leading to superstatistics," Condensed Matter Physics, vol. 9, no. 1, pp. 71-80, 2006.

[27] V. V. Ryazanov, "Lifetime distributions in the methods of nonequilibrium statistical operator and superstatistics," European Physical Journal B, vol. 72, no. 4, pp. 629-639, 2009.

[28] V. V. Ryazanov, "Statistical substantiation of introduction of the distributions containing lifetime as thermodynamic parameter," http://arxiv.org/abs/0710.2449. 

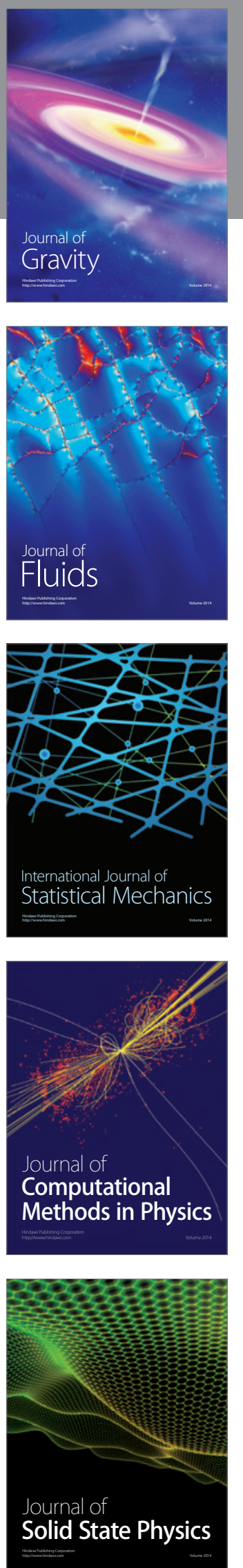

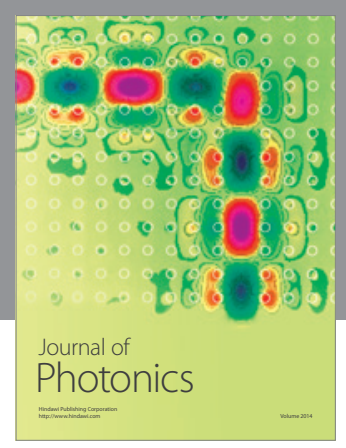

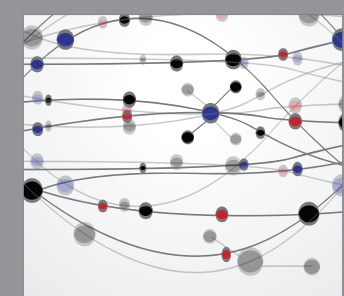

The Scientific World Journal
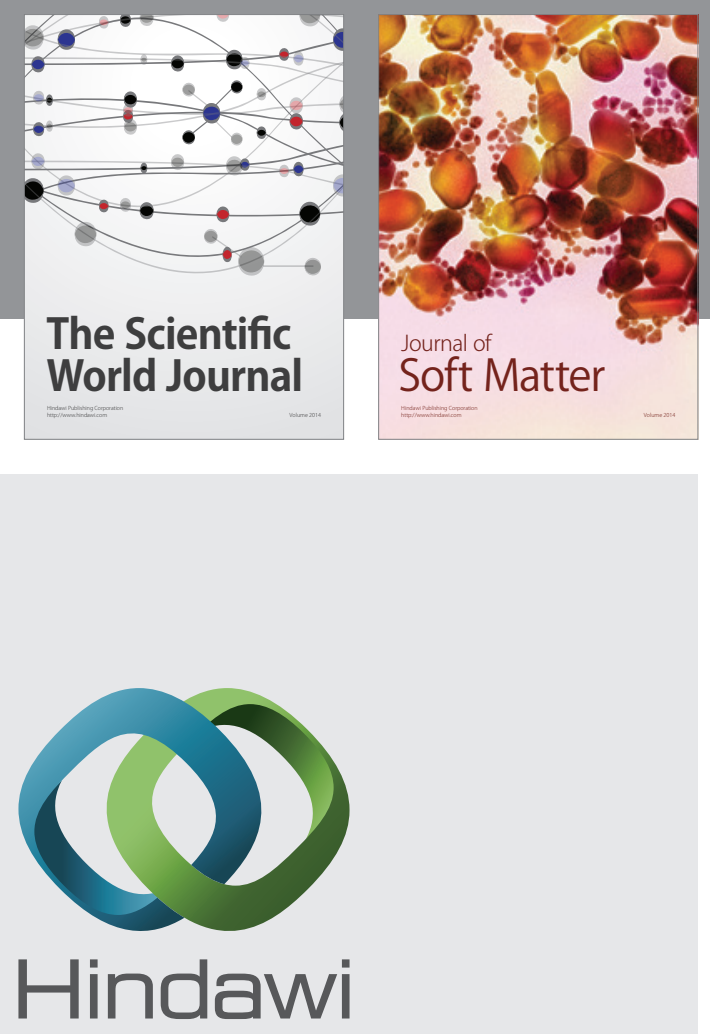

Submit your manuscripts at

http://www.hindawi.com
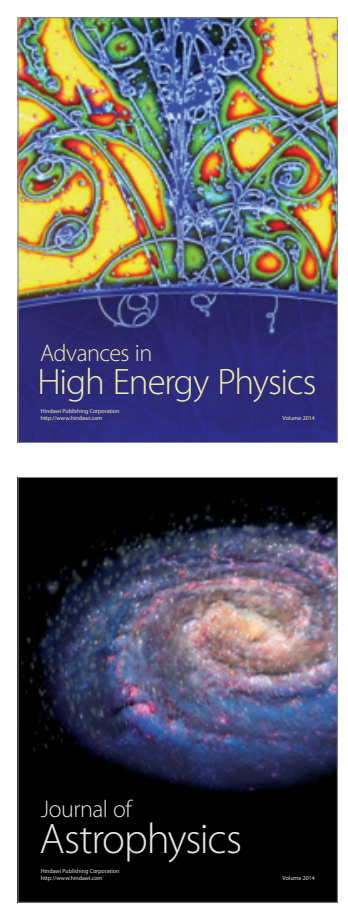
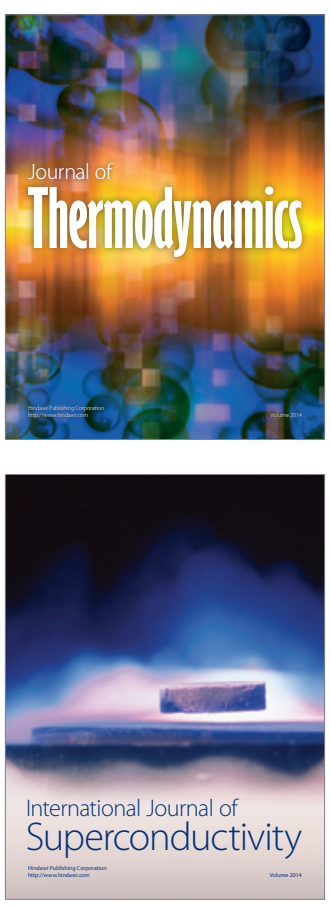
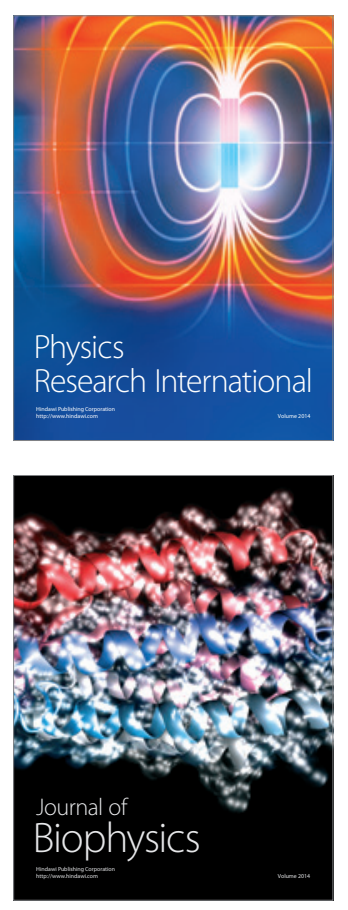
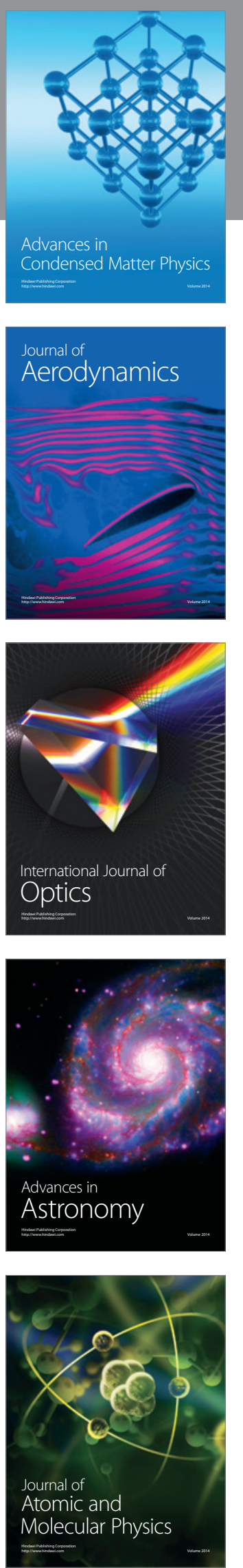